\title{
The use of patient-reported outcomes in routine cancer care: preliminary insights from a multinational scoping survey of oncology practitioners
}

\author{
Yin Ting Cheung ${ }^{1}$ - Alexandre Chan ${ }^{2} \cdot$ Andreas Charalambous $^{3,4} \cdot$ H. S. Darling ${ }^{5}$ Lawson Eng ${ }^{6} \cdot$ Lisa Grech $^{7,8,9,10}$. \\ Corina J. G. van den Hurk ${ }^{11}$. Deborah Kirk ${ }^{12}$. Sandra A. Mitchell ${ }^{13}$. Dagmara Poprawski ${ }^{14}$. Elke Rammant ${ }^{15}$. \\ Imogen Ramsey ${ }^{16} \cdot$ Margaret I. Fitch $^{17} \cdot$ Raymond J. Chan ${ }^{18} \cdot$ on behalf of the Multinational Association for \\ Supportive Care in Cancer Survivorship Study Group.
}

Received: 8 June 2021 / Accepted: 4 September 2021 / Published online: 15 September 2021

(C) The Author(s), under exclusive licence to Springer-Verlag GmbH Germany, part of Springer Nature 2021, corrected publication 2021

\begin{abstract}
Background There exists scant evidence on the optimal approaches to integrating patient-reported outcomes (PROs) in clinical practice. This study gathered oncology practitioners' experiences with implementing PROs in cancer care.

Methods Between December 2019 and June 2020, we surveyed practitioners who reported spending > 5\% of their time providing clinical care to cancer patients. Respondents completed an online survey describing their experiences with and barriers to using PROs in clinical settings.

Results In total, 362 practitioners (physicians $38.7 \%$, nurses $46.7 \%$, allied health professionals $14.6 \%$ ) completed the survey, representing 41 countries (Asia-Pacific 42.5\%, North America 30.1\%, Europe 24.0\%, others 3.3\%). One quarter (25.4\%) identified themselves as "high frequency users" who conducted PRO assessments on $>80 \%$ of their patients. Practitioners commonly used PROs to facilitate communication (60.2\%) and monitor treatment responses (52.6\%). The most commonly reported implementation barriers were a lack of technological support (70.4\%) and absence of a robust workflow to integrate PROs in clinical care (61.5\%). Compared to practitioners from high-income countries, more practitioners in low-middle income countries reported not having access to a local PRO expert $(P<.0001)$ and difficulty in identifying the appropriate PRO domains $(P=.006)$. Compared with nurses and allied health professionals, physicians were more likely to perceive disruptions in clinical care during PRO collection $(P=.001)$ as an implementation barrier.

Conclusions Only a quarter of the surveyed practitioners reported capturing PROs in routine clinical practice. The implementation barriers to PRO use varied across respondents in different professions and levels of socioeconomic resources. Our findings can be applied to guide planning and implementation of PRO collection in cancer care.
\end{abstract}

Keywords Patient-reported outcomes $\cdot$ Patient-reported outcome measures $\cdot$ PRO $\cdot$ PROM $\cdot$ Patient-centered care

YT Cheung is the first author, MI Fitch and RJ Chan are cocorresponding authors. All other authors will be listed in alphabetical order by their last names.

\author{
Margaret I. Fitch \\ marg.i.fitch@gmail.com \\ Raymond J. Chan \\ Raymond.Chan@flinders.edu.au
}

Extended author information available on the last page of the article

\section{Introduction}

Patient-reported outcomes (PROs) have become an important component of health outcome assessments to capture the patient's subjective effects of illness and treatment. PROs are broadly defined as "any report of the status of a patient's health condition that comes directly from the patient, without interpretation of the patient's response by a clinician or anyone else". [1,2] There is emerging evidence that PROs are useful as communication tools to improve symptom control and treatment response monitoring in the oncology setting [1, $3,4]$, as well as to enhance physician-patient communication. $[4,5]$ Other than adding value to patient care at the clinician 
Symptoms and Functional Status

Disease-specific symptoms $(\mathrm{n}=177)$

Physical function $(\mathrm{n}=172)$

$$
\text { Pain ( } \mathrm{n}=166)
$$

Nausea and vomiting $(\mathrm{n}=151)$

Fatigue $(\mathrm{n}=145)$

Nutritional status $(\mathrm{n}=131)$

Functional status $(\mathrm{n}=113)$

$$
\text { Mobility }(n=113)
$$

Cognitive function $(\mathrm{n}=92)$

Sexual function $(\mathrm{n}=52)$

\section{Emotional Function and Behavior}

Depression $(\mathrm{n}=136)$

Anxiety $(\mathrm{n}=133)$

Distress $(n=116)$

Overall emotional well-being $(\mathrm{n}=111)$

$\operatorname{Mood}(\mathrm{n}=78)$

Behavioral changes $(\mathrm{n}=61)$

Psychological adjustment $(n=48)$

\section{Social Functioning}

Social well-being and function $(\mathrm{n}=121)$

Social support $(\mathrm{n}=99)$

Financial impact $(\mathrm{n}=73)$

Family impact $(\mathrm{n}=73)$

Interpersonal relationships $(\mathrm{n}=61)$

\section{General Measures of Health}

Overall quality of life $(n=133)$

Overall health status $(\mathrm{n}=92)$

Disease-specific quality of life $(\mathrm{n}=77)$

\section{Care Coordination/Experiences of Care}

Medication side effects $(\mathrm{n}=115)$

Medication adherence $(n=107)$

Satisfaction with care $(n=73)$

Barriers to care $(\mathrm{n}=59)$

Information provision $(\mathrm{n}=34)$

Transition readiness $(n=25)$
Proportion (\%)
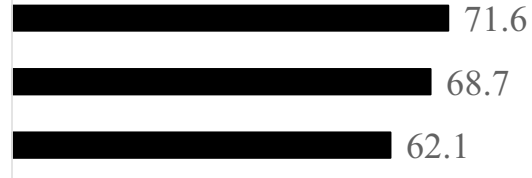

56.9

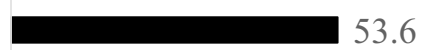

43.6

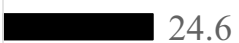

24.6
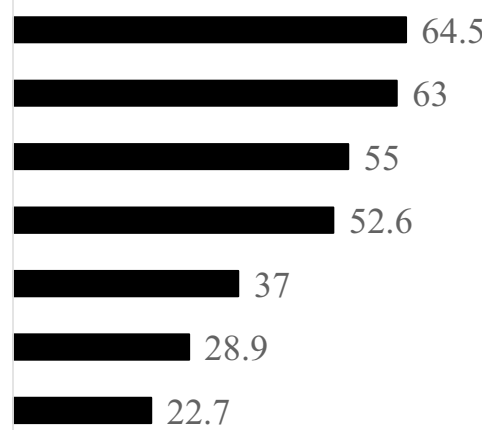

22.7
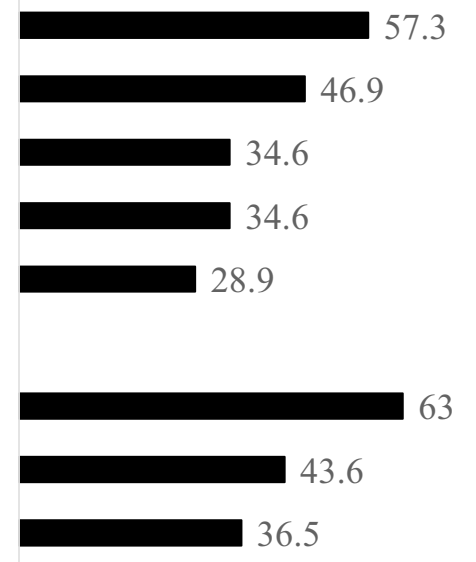
63

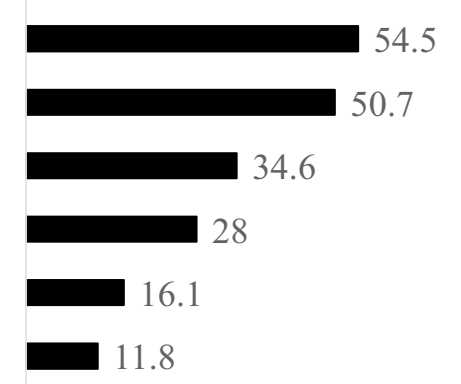


४Fig. 1 Patient-reported outcomes domains collected by respondents $(n=211)$. Analysis was conducted in the 211 respondents who indicated that they conduct patient-reported outcome collection for clinical practice. Participants could select more than one response

level, recent studies have also reported the impact of PRO monitoring on greater benefits, including longer overall survival and reduced emergency department use. [6-9] PROs are contributing to the paradigm shift to patient-centered care and system to drive improvements in healthcare quality. [10,11].

Despite the growing interests in routine PRO collection to facilitate patient-centered care in cancer settings, the adoption of PROs in routine clinical practice has challenges. First, there are concerns with the choice of a valid and reliable PRO measure, which refers to a questionnaire or tool that probes individual patient perspectives on their health and health-related experiences and outcomes. [1, 12, 13] Clinicians may experience a lack of guidance on the choice of appropriate tools to capture constructs that are meaningful to both patients and clinicians. [5, 12, 14] Data collection by using a PRO measure can be conducted through self-administration, interviews, or combination of different approaches. Consequently, the collection of PROs may add undue burden on patients who do not recognize the relevance of PRO measures or have low literacy skills.

On a macro level, technical and administrative challenges in establishing a user-friendly platform for electronic data capture, linkage with relevant clinical characteristics, and concerns about data security also hinder the successful implementation PRO monitoring in care delivery settings. [3, 15-18] Other system-level challenges include difficulties assimilating the use of PRO information into clinical workflows $[3,4,19]$ and deficiencies in the expertise needed to interpret PRO data and apply the information to clinical decision-making. [17, 20, 21].

Few studies have comparatively evaluated patterns of PRO adoption in cancer clinical practice in different regions of the world. For example, most PRO measures have been developed in high-income settings, and their applicability in low resourced settings may be limited. Furthermore, most studies are focused on discussing experiences and implementation challenges in users of PROs, and little is known about the barriers experienced by oncology practitioners who indicate they are non-users or infrequent users of PROs in clinical practice.

With the overarching aim of capturing preliminary insights into the practice patterns of oncology practitioners representing different regions and resource settings, this scoping survey captured oncology practitioners' clinical experiences with using PRO and identified barriers and facilitators to their use in the delivery of cancer care. Findings from this study may inform implementation of routine PRO surveillance and promoting patient-centered care around the world.

\section{Methods}

\section{Study design and setting}

This multinational, cross-sectional survey study was conducted between December 2019 and June 2020. Approval was obtained from the Survey and Behavioral Research Ethics Committee of the Chinese University of Hong Kong (Hong Kong; Reference number: SBRE-18-512) and the Research Ethics Board of Edith Cowan University (Australia; Reference number: 2019-00334-WALKER).

\section{Eligibility and recruitment}

Study participants were recruited using a combination of convenience and snowball sampling approaches. To be eligible for this survey, participants had to identify themselves as: (1) older than 18 years; (2) able to understand written English, (3) a practicing clinician and/or practitioner, including but not limited to a physician, nurse, or allied healthcare professional; and (4) spending at least $5 \%$ of their time providing direct clinical care to cancer patients. This set of criteria was decided a priori to include both fulltime and non-fulltime clinicians/practitioners, such as clinician scientists, academic clinicians, or clinician administrators.

\section{Survey instrument}

The development of the survey was led by the investigators for this study, which included 14 members representing North America, Europe, and Asia-Pacific practice settings. The team consists of PRO experts (CJGH, IR, RJC), survey methodologists (YTC, SAM), oncologists (HSD, LE, DP), oncology nurses (DK, MIF), and allied health professionals (AC, AC, ER). The survey was self-administered in English and took approximately $10-15 \mathrm{~min}$ to complete. A pilot study was conducted with 15 oncology practitioners to refine the survey questions and administrative procedures.

The online survey was designed based on review of the existing literature on implementation sciences regarding the use of PRO in cancer care (e.g., identifying common barriers and facilitators). [1, 3-5, 14, 16-18, 20, 21] The survey was comprised of three sections (total 40 items) and included a short introduction describing the overall objective of the study. The first section collected demographic and workrelated information. The second section focused on respondents' experiences with PRO collection in their respective clinical settings. These items assessed the frequency of PRO use in practice ( $0-100 \%$ of patients), time points for PRO data collection, PRO measures used and domains assessed (Fig. 1), and mode of PRO administration (e.g., paper-based, 
electronic tablets, mobile applications). Respondents were also asked about the downstream utility and actionability of the PRO data in supporting decision-making, informing risk stratification and prognosis, and facilitating monitoring of response to treatment. The last section included a list of 18, practitioner-, patient-, and system-related implementation barriers to the routine collection of PROs. [1, 3-5, 14, 16-18, 20, 21] Respondents rated the salience of these potential barriers on a 5-point Likert scale ranging from "totally agree" to "totally disagree." Respondents also had the option to provide additional barriers to PRO collection.

\section{Data collection}

Participants were invited to take part in the study via a survey link shared by specialist cancer organizations, professional societies, and members of the research team in emails and other forms of online communication. We took reasonable effort in ensuring that professional societies serving the major geographical regions, disciplines, and professionals were invited to participate in the survey. The final list of professional societies who facilitated the dissemination of the survey to their society members is presented in Supplement 1. Professional organizations sent a reminder approximately two weeks after the initial invitation. To begin the survey, respondents had to indicate that they fulfilled the inclusion criteria. Completion and submission of the survey implied informed consent to participate. Respondents were assured that their responses would be anonymous and only aggregated data would be reported. At the conclusion of the survey, all respondents were encouraged to disseminate the survey link to at least five other oncology practitioners who fulfilled the inclusion criteria.

The sample size is determined based on feasibility and attempt to reach as many eligible respondents as possible. It was decided a priori that each pre-defined group should have approximate 100 respondents. The pre-defined groups were based on country-level income (high-income countries [HICs] versus middle- and low-income countries [LMICs], as defined by the World Bank) and profession (nurses versus physicians and allied health professionals). These predefined groups were chosen as the existing literature has highlighted socioeconomic status and discipline as major factors influencing the uptake of PRO collection in clinical practice $[4,5,14,16,17,20]$, and that such data could be readily self-reported or inferred from a questionnaire. The main data collection phase was conducted from December 2019 to March 2020. Subsequently, the characteristics of respondents were reviewed. During the second data collection phase from April 2020 to June 2020, the survey was recirculated or specifically targeted at the underrepresented groups or sectors.

\section{Data analysis}

Participant responses were recorded anonymously and retrieved electronically using the online survey software Qualtrics (SAP). We reviewed the IP addresses to confirm that no participants submitted multiple entries. Only questionnaires with completed responses for both Sects. 1 (demographics) and 2 (experience with PRO collection) were analyzed. The Checklist for Reporting Results of Internet E-Surveys was adopted to report the online survey findings (Supplement 2). [22].

Descriptive statistics were used to summarize responses to each question. Categorical responses were compared using Chi-square test for independence between the following predefined groups: (1) physicians versus nurses versus allied health professionals; (2) HICs versus LMICs; and (3) frequency of PRO use in practice. Due to the scoping nature of the study, adjustment for multiple comparisons was not conducted. All analyses were conducted using SAS (SAS 9.4, SAS Institute, Cary, NC, USA).

\section{Results}

In total, 402 individuals responded to the survey invitation by attempting the survey, of whom 366 submitted the completed survey. After excluding responses that had missing demographic information $(n=4)$, surveys from 362 oncology practitioners were available for analysis. Sample characteristics are presented in Table 1 . The majority were female $(n=257,71.0 \%)$ and younger than 50 years of age $(n=224,61.8 \%)$. Almost half of the respondents were nurses ( $n=169,46.7 \%)$, followed by physicians $(n=140,38.7 \%$ ). The remaining respondents $(n=53,14.6 \%)$ were allied health professionals, including pharmacists, psychologists, and social workers. The majority of respondents practiced in the Asia-Pacific region $(n=154,42.5 \%)$, North America $(n=109,30.1 \%)$, Western Europe $(n=72,19.9 \%)$, Eastern Europe $(n=15,4.1 \%)$, Africa $(n=8,2.2 \%)$, and Latin America/Caribbean region $(n=4,1.1 \%)$ and represented 41 countries or administrative regions in total (Supplement 3). A majority of the respondents practiced in HICs $(n=263$, $72.7 \%)$ and had over 10 years of experience in cancer care $(n=253,69.9 \%)$.

\section{Frequency of PRO use in practice}

Approximately two-thirds of respondents used PROs in their clinical practice (Table 2), with nearly one-quarter identifying themselves as "high frequency users" who conducted PRO assessments with more than $80 \%$ of their patients $(n=91,25.4 \%)$. Another one-third were "moderate frequency users" (40-80\% of their patients; $n=80,22.3 \%$ ), 
Table 1 Demographic and clinical experience of respondents $(n=362)$

\begin{tabular}{|c|c|c|c|}
\hline & & $N$ & $\%$ \\
\hline \multirow[t]{5}{*}{ Age (years) } & $19-29$ & 25 & 6.9 \\
\hline & $30-39$ & 95 & 26.2 \\
\hline & $40-49$ & 104 & 28.7 \\
\hline & $50-59$ & 98 & 27.1 \\
\hline & Above 60 & 40 & 11.0 \\
\hline \multirow[t]{3}{*}{ Sex } & Male & 104 & 28.7 \\
\hline & Female & 257 & 71.0 \\
\hline & Prefer not to answer & 1 & 0.3 \\
\hline \multirow[t]{8}{*}{ Type of practice setting* } & Community, government, municipal hospital, or tertiary care center & 145 & 40.1 \\
\hline & Academic, university, or research centers & 121 & 33.4 \\
\hline & Comprehensive cancer centers & 115 & 31.8 \\
\hline & Hospice or end of life care & 7 & 1.9 \\
\hline & Private practice & 35 & 9.7 \\
\hline & Government organizations, ministry of health, health authorities & 8 & 2.2 \\
\hline & Non-governmental organization or patient support group & 8 & 2.2 \\
\hline & Others & 4 & 1.1 \\
\hline \multirow[t]{6}{*}{ Geographical region } & Africa & 8 & 2.2 \\
\hline & Asia-Pacific & 154 & 42.5 \\
\hline & Eastern Europe & 15 & 4.1 \\
\hline & Latin America and Caribbean & 4 & 1.1 \\
\hline & North America & 109 & 30.1 \\
\hline & Western Europe & 72 & 19.9 \\
\hline \multirow[t]{2}{*}{ Income level } & Low-middle income countries & 99 & 27.3 \\
\hline & High-income countries & 263 & 72.7 \\
\hline \multirow[t]{16}{*}{ Profession } & Physician: & 140 & 38.7 \\
\hline & Medical oncologist & 94 & 67.6 \\
\hline & Family practice & 2 & 1.4 \\
\hline & General medicine & 3 & 2.2 \\
\hline & Radiation oncologist & 11 & 7.9 \\
\hline & Hematologist & 7 & 5.0 \\
\hline & Surgeons & 14 & 10.1 \\
\hline & Others & 8 & 5.8 \\
\hline & Nurse & 169 & 46.7 \\
\hline & Allied health professionals: & 53 & 14.6 \\
\hline & Oral hygienists & 11 & \\
\hline & Pharmacist & 21 & \\
\hline & Rehabilitation specialist & 5 & \\
\hline & Speech therapist & 5 & \\
\hline & Physiotherapist & 4 & \\
\hline & Others & 7 & \\
\hline
\end{tabular}


Table 1 (continued)

\begin{tabular}{|c|c|c|c|}
\hline & & $N$ & $\%$ \\
\hline \multirow[t]{15}{*}{ Primary areas of cancer practice* } & All cancer types & 165 & 45.6 \\
\hline & Head and neck & 84 & 23.2 \\
\hline & Breast & 83 & 22.9 \\
\hline & Gastrointestinal/colorectal & 80 & 22.1 \\
\hline & Lung & 73 & 20.2 \\
\hline & Gynecologic & 70 & 19.3 \\
\hline & Palliative oncology & 68 & 18.8 \\
\hline & Genitourinary & 59 & 16.3 \\
\hline & Hematological & 58 & 16.0 \\
\hline & Lymphomas/myeloma & 55 & 15.2 \\
\hline & Survivorship/rehabilitation & 32 & 8.8 \\
\hline & Pediatric and AYA cancers & 20 & 5.5 \\
\hline & Brain/central nervous system & 10 & 2.8 \\
\hline & Sarcoma & 10 & 2.8 \\
\hline & Others & 10 & 2.8 \\
\hline \multirow[t]{8}{*}{ Years of clinical experience } & Less than a year & 4 & 1.1 \\
\hline & $1-4$ years & 46 & 12.7 \\
\hline & $5-9$ years & 59 & 16.3 \\
\hline & $10-14$ years & 67 & 18.5 \\
\hline & $15-19$ years & 48 & 13.3 \\
\hline & $20-24$ years & 41 & 11.3 \\
\hline & $25-29$ years & 38 & 10.5 \\
\hline & 30 years or above & 59 & 16.3 \\
\hline
\end{tabular}

*Multiple responses allowed

or "low frequency users" (less than $40 \%$ of their patients; $n=40,11.2 \%$ ). A notable proportion of oncology practitioners indicated that they did not utilize any PRO collection in their clinical setting $(n=123,34.4 \%)$ or were uncertain whether their setting employed routine PRO collection in clinical practice $(n=24,6.7 \%)$. No difference in the frequency of PRO use in practice was identified among different professions $(P=0.68)$ or between HICs and LMICs $(P=0.89)$ (Supplement 4).

\section{Experience with PRO collection}

Among the practitioners who reported that they used PROs in their clinical practice ( $n=211,58.9 \%$ ), the majority collected PROs from all patients irrespective of cancer type ( $n=183,86.7 \%$ ) or place on the cancer care continuum ( $n=184,87.6 \%$ ) (Table 2). Most oncology practitioners indicated that PRO assessments were solicited using traditional "paper-based" methods ( $n=132,62.6 \%)$, verbally through clinician interviews ( $n=96,45.5 \%)$, or using electronic tablets and/or computers $(n=95,45 \%)$.

The most commonly collected PRO domains were disease- and treatment-specific symptoms (Fig. 1). The least commonly collected domains were those related to care coordination and patient experiences of care, including transition readiness and information provision.

Oncology practitioners reported using PRO data to facilitate communication with patients $(n=127,60.2 \%)$, monitor general health status $(n=114,54.0 \%)$, and responses to treatment ( $n=111,52.6 \%$ ) (Table 2$)$. Fewer respondents reported using PRO data to set treatment goals $(n=94$, $44.5 \%)$ or facilitate communication among healthcare professionals $(n=69,32.7 \%)$.

\section{Barriers to implementing PRO collection}

The most common practitioner-related barriers to PRO collection were inadequate access to a local PRO expert $(53.4 \%)$ and uncertainty about how to select an 
Table 2 Experience with patient-reported outcome collection

\begin{tabular}{|c|c|c|c|}
\hline & & $N$ & $\%$ \\
\hline \multirow[t]{7}{*}{ Frequency of practice } & High frequency users ( $80 \%$ to $100 \%$ of patients) & 91 & 25.4 \\
\hline & Moderate frequency users ( $60 \%$ to $80 \%$ of patients): & 80 & 22.3 \\
\hline & $40 \%$ to $60 \%$ of patients & 48 & 13.4 \\
\hline & $40 \%$ to $60 \%$ of patients & 32 & 8.9 \\
\hline & Low frequency users (less than $40 \%$ of patients) & 40 & 11.2 \\
\hline & Never-users & 123 & 34.4 \\
\hline & Not sure & 24 & 6.7 \\
\hline \multirow[t]{14}{*}{ Target patients* ${ }^{\mathrm{f}}$} & All cancer types & 183 & 86.7 \\
\hline & Only specific cancer types: & 28 & 13.3 \\
\hline & Blood cancers & 3 & 10.7 \\
\hline & Brain & 1 & 3.6 \\
\hline & Breast & 10 & 35.7 \\
\hline & Prostate & 1 & 3.6 \\
\hline & Colorectal & 2 & 7.1 \\
\hline & Lung & 2 & 7.1 \\
\hline & Head and neck & 6 & 21.4 \\
\hline & Gynecological cancers & 5 & 17.9 \\
\hline & Melanoma & 2 & 7.1 \\
\hline & Neuroendocrine neoplasms & 1 & 3.6 \\
\hline & Genitourinary & 1 & 3.6 \\
\hline & Thyroid & 1 & 3.6 \\
\hline \multirow[t]{7}{*}{ Time points of PRO collection* } & All time points & 184 & 87.6 \\
\hline & Only specific time points: & 26 & 12.4 \\
\hline & At diagnosis & 16 & 61.5 \\
\hline & During active treatment & 15 & 57.7 \\
\hline & Early phase of follow-up ( $<2$ years from completion of treatment $)$ & 14 & 53.8 \\
\hline & Long-term follow-up ( $>2$ years from completion of treatment) & 6 & 23.1 \\
\hline & Palliative & 2 & 7.7 \\
\hline \multirow[t]{6}{*}{ Mode of data collection* ${ }^{\mathrm{f}}$} & "Paper and pen" & 132 & 62.6 \\
\hline & Collected by healthcare providers & 77 & 36.5 \\
\hline & Electronic tablets/ computers & 95 & 45.0 \\
\hline & Mobile application & 22 & 10.4 \\
\hline & Wearable devices & 4 & 1.9 \\
\hline & Interview (verbally collected) & 96 & 45.5 \\
\hline \multirow[t]{14}{*}{ Goals of PRO collection* ${ }^{\mathrm{f}}$} & Facilitating monitoring: & & \\
\hline & Patient's general health status & 114 & 54.0 \\
\hline & Response to treatment/management & 111 & 52.6 \\
\hline & Supporting decision-making in: & & \\
\hline & Diagnostic and screening processes & 106 & 50.2 \\
\hline & Indication for treatment & 126 & 59.7 \\
\hline & Risk stratification and prognosis & 66 & 31.3 \\
\hline & Setting of treatment goals & 94 & 44.5 \\
\hline & $\begin{array}{l}\text { Model of follow-up care (e.g., oncology specialist care vs. general practitioner-led } \\
\text { vs. nurse-led vs. self-management) }\end{array}$ & 66 & 31.3 \\
\hline & Facilitating communication: & & \\
\hline & Between patients and health professionals & 127 & 60.2 \\
\hline & Within teams and between professionals & 69 & 32.7 \\
\hline & Research & 75 & 35.5 \\
\hline & Not sure & 7 & 3.3 \\
\hline
\end{tabular}

*Analysis was conducted in the 211 respondents who indicated that they conduct patient-reported outcome collection in their clinical settings

${ }^{\mathrm{I}}$ Multiple responses allowed 
appropriate PRO measure (53.4\%) (Fig. 2). Only a minority of practitioners thought that PROs did not play an important role in clinical decision-making $(8.7 \%)$. However, respondents endorsed several patient-level barriers to PRO collection including low literacy (40.2\%), a perception that PRO collection was burdensome (32.1\%), or that patients were unwilling to adhere to periodic PRO reporting $(21.2 \%)$. With respect to system-related barriers, the majority of practitioners highlighted a lack of technological support (70.4\%) and staff support (64.8\%) and the absence of a robust clinical workflow that integrated PRO reporting (61.5\%). Limited time to collect PROs during clinical interactions $(46.0 \%)$ and challenges in ensuring that PRO and trend data are available to practitioners in real-time (37.0\%) were other common barriers. A minority of the respondents $(n=6)$ provided additional barriers such as the lack of support from the management level, lack of cost-effectiveness data, PROs not consistently captured across clinics within an institution, and difficulty in differentiating PROs for research versus clinical uses.

\section{Comparisons of barriers between LMICs and HICs}

Distinct barriers were identified by respondents from LMICs and HICs (Table 3). Compared with practitioners who worked in HICs, a greater proportion of practitioners in LMICs reported that they did not have access to a local PRO expert (76.3\% versus $44.8 \%, P<0.0001$ ) or experienced difficulty in selecting meaningful PRO domains to measure $(50.5 \%$ versus $33.3 \%, P=0.006)$. Compared to those in HICs, respondents in LMICs were also more concerned about the impact of patient non-adherence to PRO reporting PROs (34.0\% versus $16.5 \%, P=0.001)$ and low literacy levels $(59.8 \%$ versus $33.0 \%, P<0.0001)$. The same trends comparing LMICs with HICs were also seen with respect to system level barriers, including inadequate staff support $(P=0.009)$, the absence of a robust clinical workflow for PRO integration $(P=0.006)$, and difficulty in accessing PRO trend data in real-time $(P=0.032)$.

\section{Comparisons of barriers among physicians, nurses, and allied health professionals}

Compared with nurses and allied health professionals (Table 3), physicians were more likely to acknowledge challenges with PRO collection in terms of manpower shortage $(P=0.003)$, poor technical support $(P=0.002)$, disruptions in clinical workflow during PRO collection $(P=0.001)$, lack of an effective workflow $(P<0.0001)$, and not having enough time during clinical interactions to collect PROs $(P<0.001)$.

\section{Comparisons of frequency of PRO use in practice}

High-frequency users of PROs reported the fewest barriers, as compared with moderate- and low-frequency users and never-users of PROs (Supplement 5). These differences were consistently observed for multiple practitioner-related, patient-related, and system-related barriers.

\section{Discussion}

This survey study aimed to gather oncology practitioners' experiences with PRO data collection for clinical care. Despite the study being conducted during the pandemic on a modest sample size of respondents, this scoping study is the first to provide preliminary insights about the experiences and perspectives of oncology practitioners at a global level. While the vast majority of oncology practitioners acknowledged the important role of PROs in clinical practice, more than half were either low-frequency users or never-users of PROs. The majority of the respondents' application of PROs was to facilitate physician-patient communication and monitoring health status. Only a minority reported integrating the PROs with clinician feedback and trigger tools.

The knowledge gaps around selection of PROs for clinical practice were the most prevalent practitioner-related barrier to implementing PRO assessment in clinical practice, particularly for physicians. The literature offers numerous validated PRO measures that are readily available for use. [23, 24] However, the majority of guidelines on the selection of PRO measures were designed for clinical trials and research, which may well explain the lack of guidance suited to practitioners and in the real-world clinical setting. To address this gap, several groups, such as the International Society for Quality of Life Research and the Patient-Centered Outcomes Research Institute, are now developing systematic, evidencebased approaches to select, implement, and evaluate PROMs in the clinical setting. [13, 15, 25-27] There are also a growing number of core outcome sets recommended by the International Consortium for Health Outcomes Measurement $[28,29]$, which reflect expert consensus on the minimum PROs to measure for a given outcome and context. These are intended to facilitate the process of outcome selection by recommending domains and measures that are considered important by patients and clinicians.

As for barriers at the organizational or systems level, our respondents consistently highlighted the lack of technological support and the need for a robust workflow to integrate PRO into clinical management. The inability to retrieve and trend PRO information in real-time for consultation also posed a challenge for the majority of respondents. Undeniably, the solutions to address these barriers differ across 
Fig. 2 Barriers to implementation of patient-reported outcome collection in clinical practice $(n=362)$. PRO, patient-reported outcomes

\section{Practitioner-related Barriers}

Do not have access to a local PRO expert

Unsure about selecting a PRO measure

Unsure about what domains to measure

Unsure about applying PRO to clinical decisions

Concerns about medico-legal liability

Do not recognize the value of PRO

\section{Patient-related Barriers}

Low literacy levels

PRO collection too burdensome

Non-participation in PRO reporting

Do not recognize the value of PRO

Concerns with confidentiality issues

Too ill to report PRO

\section{System-related Barriers}

Lack of technological support

Lack of staff support

Lack of a robust workflow to integrate PRO

Not enough time during clinical interactions

Difficulty in retrieving real time PRO data

Do not recognize the role of PRO

Concerns with disruption of workflow

Concerns with privacy and security

$$
0 \%
$$

\section{4 \\ 16.8 \\ 29.8}

\begin{tabular}{|l|l|l|}
\hline 43.6 & 16.2 & 40.2 \\
\hline 38.0 & 17.6 & 44.4 \\
\hline
\end{tabular}

\begin{tabular}{|l|l|l|}
\hline 33.2 & 19.0 & 47.8 \\
\hline
\end{tabular}

\begin{tabular}{|l|l|l|}
\hline 21.5 & 33.5 & 45.0 \\
\hline
\end{tabular}

\begin{tabular}{|l|l|}
\hline 8.710 .9 & 80.4 \\
\hline
\end{tabular}

\begin{tabular}{|l|l|l|}
\hline 40.2 & 19.6 & 40.2 \\
\hline \multicolumn{3}{|c|}{} \\
\hline 32.1 & 32.7 & 35.2 \\
\hline
\end{tabular}

\begin{tabular}{l|l|l|}
\hline 21.2 & 23.2 & 55.6 \\
\hline
\end{tabular}

\begin{tabular}{l|l|l}
20.9 & 27.4 & 51.7
\end{tabular}

\begin{tabular}{|l|l|l|}
\hline 19 & 29.9 & 51.1 \\
\hline
\end{tabular}

\begin{tabular}{ll|l}
\hline 14.0 & 13.7 & 72.3
\end{tabular}

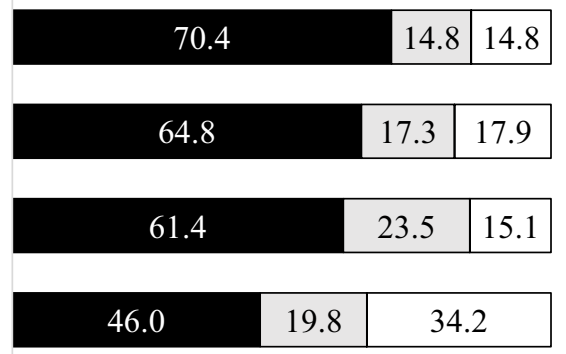

\section{0} 35.3 27.7

\begin{tabular}{|l|l|l|}
\hline 34.6 & 22.1 & 43.3 \\
\hline
\end{tabular}

\section{8}

$21.2 \quad 45.0$

26.3

\begin{tabular}{l|l}
27.4 & 46.3
\end{tabular}

$0 \%$

घ Strongly Agree/ Agree $\square$ Neutral $\square$ Disagree/ Strongly Disagree 
Table 3 Comparison of barriers to implementing patient-reported outcome measures by regions of income levels and profession

\begin{tabular}{|c|c|c|c|c|c|c|c|}
\hline & \multicolumn{3}{|c|}{ Income level } & \multicolumn{4}{|l|}{ Profession } \\
\hline & $\begin{array}{l}\text { LMIC } \\
(n=97)\end{array}$ & $\begin{array}{l}\text { HIC } \\
(n=261)\end{array}$ & $P$ & $\begin{array}{l}\text { Physicians } \\
(n=139)\end{array}$ & $\begin{array}{l}\text { Nurses } \\
(n=163)\end{array}$ & $\begin{array}{l}\text { AH Professionals } \\
(n=52)\end{array}$ & $P$ \\
\hline Practitioner-related barriers & $n(\%)$ & $n(\%)$ & $n(\%)$ & $n(\%)$ & $n(\%)$ & $n(\%)$ & \\
\hline Do not have access to a local PRO expert & $74(76.3)$ & $117(44.8)$ & $<.0001$ & $84(60.4)$ & $78(47.9)$ & $27(51.9)$ & 0.004 \\
\hline Unsure about selecting a PRO measure & $47(48.5)$ & $109(41.8)$ & 0.14 & $62(44.6)$ & $68(41.7)$ & $25(48.1)$ & 0.67 \\
\hline Unsure about what domains to measure & $49(50.5)$ & $87(33.3)$ & 0.006 & $66(47.5)$ & $50(30.7)$ & $19(36.5)$ & 0.058 \\
\hline Unsure about applying PRO in clinical decisions & $32(33.0)$ & $87(33.3)$ & 0.89 & $48(34.5)$ & $55(33.7)$ & $15(28.8)$ & 0.88 \\
\hline Concerns with liability issues & $26(26.8)$ & $51(19.5)$ & 0.10 & $30(21.6)$ & $34(20.9)$ & $12(23.1)$ & 0.49 \\
\hline Do not recognize the role of PRO & $8(8.2)$ & $23(8.8)$ & 0.10 & $8(5.8)$ & $14(8.6)$ & $7(13.5)$ & 0.24 \\
\hline \multicolumn{8}{|l|}{ Patient-related barriers } \\
\hline Low health literacy level & $58(59.8)$ & $86(33.0)$ & $<.0001$ & $62(44.6)$ & $56(34.4)$ & $25(48.1)$ & 0.24 \\
\hline PRO collection too burdensome & $29(29.9)$ & $86(33.0)$ & 0.48 & $53(38.1)$ & $45(27.6)$ & $17(32.7)$ & 0.31 \\
\hline Not adherent with reporting PRO & $33(34.0)$ & $43(16.5)$ & 0.001 & $37(26.6)$ & $24(14.7)$ & $15(28.8)$ & 0.065 \\
\hline Do not recognize the role of PRO & $25(25.8)$ & $50(19.2)$ & 0.28 & $38(27.3)$ & $27(16.6)$ & $75(21.2)$ & 0.050 \\
\hline Concerns with confidentiality issues & $28(28.9)$ & $40(15.3)$ & 0.005 & $32(23.0)$ & $24(14.7)$ & $12(23.1)$ & 0.11 \\
\hline Too ill to report PRO & $15(15.5)$ & $35(13.4)$ & 0.10 & $18(12.9)$ & $23(14.1)$ & $9(17.3)$ & 0.81 \\
\hline \multicolumn{8}{|l|}{ Institution-related barriers } \\
\hline Lack of technological support & $77(79.4)$ & $175(67.0)$ & 0.052 & $115(82.7)$ & $101(62.0)$ & $33(63.5)$ & 0.002 \\
\hline Lack of staff support & $75(77.3)$ & $157(60.2)$ & 0.009 & $107(77.0)$ & $90(55.2)$ & $32(61.5)$ & 0.003 \\
\hline Lack of a robust workflow to integrate PRO & $72(74.2)$ & $148(56.7)$ & 0.006 & $104(74.8)$ & $82(50.3)$ & $31(59.6)$ & $<.0001$ \\
\hline Not enough time during clinical interactions & $56(57.7)$ & 107 (41.6) & 0.015 & $83(59.7)$ & $61(37.7)$ & $18(36.7)$ & $<.001$ \\
\hline Difficulty in retrieving real-time PRO data & $46(47.9)$ & $81(32.8)$ & $\mathbf{0 . 0 3 2}$ & $64(46.7)$ & $45(28.1)$ & $16(37.2)$ & 0.009 \\
\hline Do not recognize the role of PRO & $38(39.2)$ & $86(33.0)$ & 0.46 & $55(39.6)$ & $45(27.6)$ & $22(42.3)$ & 0.13 \\
\hline Concerns with disruption of workflow & $41(42.3)$ & $80(30.7)$ & 0.10 & $63(45.3)$ & $39(23.9)$ & $19(36.5)$ & 0.001 \\
\hline Concerns with privacy and security & $38(39.2)$ & $56(21.5)$ & $<.0001$ & $47(33.8)$ & $31(19.0)$ & $15(28.8)$ & 0.054 \\
\hline
\end{tabular}

Boldface indicates statisticalsignificance $(P<0.05)$

$A H$ allied health, $H I C$ high-income countries, $L M I C$ low-middle income countries, $P R O$ patient-reported outcome.

care delivery contexts and require engagement from multiple stakeholders, including those in management roles. We recommend that organizations identify a local expert, establish clinical champions,and/or care a working group to set realistic and consensus-driven expectations for the successful implementation of PROs. [30, 31] For example, institutions that are still in the early phases of implementation can focus on monitoring and adjusting algorithms to optimize the likelihood of success. To address the issues of limited staff support and resources, they may also consider adopting a staggered or waved rollout using principles of implementation science. These principles include developing specific steps for implementation, linking PROs to referral pathways and other tools to support clinical decision-making, and piloting the protocol on a manageable sample of patients using small cycles of change. [15] In high-resource settings with mature information technology systems, efforts should be geared toward refining the optimal features and optimizing the functionality of integrated PRO systems in clinical workflows. For example, using mobile- and tablet-based applications and wearable computing devices to collect PROs may allow remote monitoring of patients' responses throughout the course of care and reduce administration time during the clinical encounter.

Previous studies have noted that in many developing countries, clinicians typically make treatment decisions unilaterally rather than systematically involving patients in shared decision-making and care planning. [32-35] It is therefore encouraging that the majority of the respondents, in both LMIC and HIC, affirmed the important role and value of including PROs in clinical care. Rather, more respondents from LMICs than from HICs were concerned about the lack of access to expertise and knowledge on collecting PROs, as well as patients' low literacy and poor adherence to reporting PROs. One implication of this finding is the need to consider the unique context and priorities of a care delivery setting when implementing and scaling programs for PRO measurement in clinical practice. For example, given that improving survival rates and access to effective treatments remain the primary goal for healthcare systems in LMICs, patients may still appreciate the relevance of reporting PROs that are deemed useful at the point 
of care, such as disease-specific symptoms and financial toxicity.

With regard to developing specific strategies for LMICs and countries with low uptake of PROs, we recommend stakeholder engagement to strengthen the inclusion of PROs in the delivery of cancer care, as well as the translation, cultural adaptation, and validation of PRO measures for the local population. It may also be important to consider equity and disparity in access to PROs and subsequent implications. Other practical ways to encourage routine PRO collection in resource-limited settings are to select brief PRO measures in the patient's native language and to adopt inexpensive administration methods to reduce the cost associated with implementation. Incorporating branching logic and adaptive response functions into the PRO instruments [23, 36] may also help to identify individuals who need more detailed assessments; this approach may potentially limit survey burden, minimize administration time, and improve patient receptivity. [37] In this regard, HICs are in an excellent position to share resources, such as PRO experts, translated tools, and patient education materials, with LMICs.

Our study results should be considered in light of some limitations that may limit the generalizability of our results. Despite the multinational sampling frame and sequential approach to reach under-represented sectors, respondents from certain geographical regions, especially developing countries, were difficult to recruit most likely due to language barriers and limited access to the internet for survey completion. Sampling bias could not avoided from our convenience sampling approach, as most respondents were recruited through North American, European, and Australian professional bodies that were affiliated with the study investigators. We also expected practitioners from lower resource settings to have poorer participation in such professional societies. Even though we explicitly stated in the email invitation that respondents did not have to be using PROs currently in routine care to participate in the survey, the convenience sampling approach might still have introduced selection bias toward those using PROs in clinical practice. Considering that this is a multinational survey, the relatively small sample size might also be attributable to the untimely recruitment period, which coincided with the coronavirus disease 2019 pandemic. Our sample of respondents was heterogeneous and might not fully reflect the characteristics of the oncology workforce in all participating sites and countries. Future studies may investigate specific barriers in individual groups of healthcare professionals and in samples that are more consistently representative. For example, we found that physicians were more likely to perceive time and disruption to clinical workflow as implementation barriers. It would be meaningful to engage physicians in more indepth studies to identify recommendations for best practices with respect to administration and interpretation in clinical practice, and tools to support integration into clinical workflow. Lastly, we acknowledge concerns with type I errors as a wide range of analyses was conducted to identify barriers across the pre-defined groups without adjustment for multiple testing. Despite these limitations, this study is the first to provide preliminary insights about the experiences and perspectives of oncology practitioners at a global level. The findings from this scoping study will be critical to inform research agenda, build capacity, and shape implementation efforts toward widespread implementation of PROs in the delivery of cancer care.

\section{Conclusion}

Globally, the inclusion of PROs to improve the patient-centeredness of cancer care is still in an early stage of adoption. We identified various implementation barriers to PRO use across respondents in different professions and settings with differing levels of socioeconomic resources. These results may be used to develop implementation strategies and capacity building that supports routine inclusion of PRO reporting in cancer care settings. International collaborations and partnerships between high- and low-resource settings are highly recommended to strengthen the sharing of best implementation strategies. Local champions may be identified to serve as advocates to build capacity and promote the benefits of including PROs in routine clinical care. Future work should also engage the efforts of leaders and managers in clinical care settings and explore how PROs collected in clinical practice can also be used for population-level analytics for downstream appraisals of the quality and efficiency of the accountable healthcare-providing entity.

Supplementary Information The online version contains supplementary material available at https://doi.org/10.1007/s00520-021-06545-7.

Acknowledgements We would like to acknowledge the participating organizations listed in Supplement 1. Raymond J Chan receive salary support from the National Health and Research Council (APP1194051).

Author contribution All authors contributed to the study conception, study design, and acquisition of data. Yin Ting Cheung and Raymond $\mathbf{J}$ Chan performed data analysis and prepared the first draft of the manuscript. All authors commented on previous versions of the manuscript. All authors read and approved the final manuscript.

Data availability The data that support the findings of this study are available from the first author (Yin Ting Cheung) and corresponding author (Raymond J Chan) upon reasonable request.

Code availability Not applicable 


\section{Declarations}

Ethics approval Approval was obtained from the Survey and Behavioral Research Ethics Committee of the Chinese University of Hong Kong (Hong Kong; Reference number: SBRE-18-512) and the Research Ethics Board of Edith Cowan University (Australia; Reference number: 2019-00334-WALKER).

Consent to participate Completion and submission of the survey implied informed consent to participate.

\section{Consent for publication Not applicable}

Conflict of interest Corina J. G. van den Hurk received funding on projects involving implementation of symptom monitoring in lung and renal cell cancer from Astra Zeneca, BMS Pharma, Boehringer Ingelheim, Merck, Pfizer and Ipsen. All other authors do not have any conflict of interest.

\section{References}

1. Howell D, Molloy S, Wilkinson K et al (2015) Patient-reported outcomes in routine cancer clinical practice: a scoping review of use, impact on health outcomes, and implementation factors. Ann Oncol 26(9):1846-1858

2. Weldring T, Smith SMS (2013) Patient-reported outcomes (PROs) and patient-reported outcome measures (PROMs). Health Serv Insights 6:61-68

3. Graupner C, Breukink SO, Mul S, Claessens D, Slok AHM, Kimman ML (2021) Patient-reported outcome measures in oncology: a qualitative study of the healthcare professional's perspective. Support Care Cancer 29(9):5253-5261

4. Greenhalgh J, Dalkin S, Gooding K et al (2017) Functionality and feedback: a realist synthesis of the collation, interpretation and utilisation of patient-reported outcome measures data to improve patient care. Southampton (UK): NIHR Journals Library. (Health Services and Delivery Research, No. 5.2.) Available from: https:// www.ncbi.nlm.nih.gov/books/NBK409450/, https://doi.org/10. 3310/hsdr05020

5. Boyce MB, Browne JP, Greenhalgh J (2014) The experiences of professionals with using information from patient-reported outcome measures to improve the quality of healthcare: a systematic review of qualitative research. BMJ Qual Saf 23(6):508-518

6. Absolom K, Warrington L, Hudson E et al (2021) Phase III randomized controlled trial of eRAPID: eHealth intervention during chemotherapy. J Clin Oncol 39(7):734-747

7. Basch E, Deal AM, Dueck AC et al (2017) Overall survival results of a trial assessing patient-reported outcomes for symptom monitoring during routine cancer treatment. JAMA 318(2):197-198

8. Basch E, Deal AM, Kris MG et al (2016) Symptom monitoring with patient-reported outcomes during routine cancer treatment: a randomized controlled trial. J Clin Oncol 34(6):557-565

9. Denis F, Basch E, Septans A et al (2019) Two-year survival comparing web-based symptom monitoring vs routine surveillance following treatment for lung cancer. JAMA 321(3):306-307

10. Lloyd H, Wheat H, Horrell J et al (2018) Patient-reported measures for person-centered coordinated care: a comparative domain map and web-based compendium for supporting policy development and implementation. J Med Internet Res 20(2):e54-e54

11. Øvretveit J, Zubkoff L, Nelson EC, Frampton S, Knudsen JL, Zimlichman E (2017) Using patient-reported outcome measurement to improve patient care. Int J Qual Health Care 29(6):874-879
12. Foster A, Foster A, Croot L et al (2018) The facilitators and barriers to implementing patient reported outcome measures in organisations delivering health related services: a systematic review of reviews. J Patient Rep Outcomes 2(1):1-16

13. Nicole M, Doris H, Zahra I et al (2020) Selecting, implementing and evaluating patient-reported outcome measures for routine clinical use in cancer: the Cancer Care Ontario approach. J Patient Rep Outcomes 4(1):101

14. Duncan EAS, Murray J (2012) The barriers and facilitators to routine outcome measurement by allied health professionals in practice: a systematic review. BMC Health Serv Res 12:96

15. Basch E, Barbera L, Kerrigan CL, Velikova G (2018) Implementation of patient-reported outcomes in routine medical care. Am Soc Clin Oncol Educ Book 38:122-134

16. Nguyen H, Butow P, Dhillon H, Sundaresan P (2021) A review of the barriers to using patient-reported outcomes (PROs) and patient-reported outcome measures (PROMs) in routine cancer care. J Med Radiat Sci 68(2):186-195

17. van der Wees PJ, Nijhuis-Van Der Sanden MW, Ayanian JZ, Black N, Westert GP, Schneider EC (2014) Integrating the use of patient-reported outcomes for both clinical practice and performance measurement: views of experts from 3 countries. Milbank Q 92(4):754-75

18. Yang L, Yang L, Manhas D et al (2018) Patient-reported outcome use in oncology: a systematic review of the impact on patient-clinician communication. Support Care Cancer 26(1):41-60

19. Nic Giolla Easpaig B, Tran Y, Bierbaum M et al (2020) What are the attitudes of health professionals regarding patient reported outcome measures (PROMs) in oncology practice? A mixedmethod synthesis of the qualitative evidence. BMC Health Serv Res 20(1):102

20. Brundage MD, Snyder CF (2012) Patient-reported outcomes in clinical practice: using standards to break down barriers. Clin Investig 2(4):343-346

21. Selby P, Velikova G (2018) Taking patient reported outcomes centre stage in cancer research - why has it taken so long? Res Involv Engagem 4(1): 1-5

22. Eysenbach G (2004) Improving the quality of web surveys: the checklist for reporting results of internet E-surveys (CHERRIES). J Med Internet Res 6(3):e34

23. National Institutes of Health. PROMIS $®$ (patient-reported outcomes measurement information system. URL: http://www.healt hmeasures.net/explore-measurement-systems/promis. Accessed 15 Feb 2021

24. Williams K, Sansoni J, Morris D, Grootemaat P, Thompson C (2016) Patient-reported outcome measures: Literature review. Australian Commission on Safety and Quality in Health Care, Sydney

25. International society for quality of life research (prepared by Aaronson N, Elliott T, Greenhalgh J, Halyard M, Hess R, Miller D, Reeve B, Santana M, Snyder C). User's guide to implementing patient-reported outcomes assessment in clinical practice, version: January 2015. URL: https://Www.isoqol.org/wp-content/uploads/ 2019/09/2015UsersGuide-Version2.pdf. Accessed 15 Feb 2021

26. Ali J, Basch E, Baumhauer J, et al (2017) Users' guide to integrating patient-reported outcomes in electronic health records. Johns Hopkins University. URL: http://eprints.whiterose.ac.uk/118679. Accessed 15 Feb 2021

27. Chan EKH, Edwards TC, Haywood K et al (2019) Implementing patient-reported outcome measures in clinical practice: a companion guide to the ISOQOL user's guide. Qual Life Res 28(3):621-627

28. Mak KS, van Bommel Annelotte C. M, Stowell C et al (2016) Defining a standard set of patient-centred outcomes for lung cancer. Eur Respir J 48(3):852-60 
29. Zerillo JA, Schouwenburg MG, van Bommel ACM et al (2017) An international collaborative standardizing a comprehensive patientcentered outcomes measurement set for colorectal cancer. JAMA Oncol 3(5):686-694

30. Ahmed S, Barbera L, Bartlett SJ et al (2020) A catalyst for transforming health systems and person-centred care: Canadian national position statement on patient-reported outcomes. Curr Oncol 27(2):90-99

31. Stover AM, Haverman L, van Oers HA et al (2020) Using an implementation science approach to implement and evaluate patient-reported outcome measures (PROM) initiatives in routine care settings. Qual Life Res. https://doi.org/10.1007/ s11136-020-02564-9

32. Couet N, Desroches S, Robitaille H et al (2015) Assessments of the extent to which health-care providers involve patients in decision making: a systematic review of studies using the OPTION instrument. Health Expect 18(4):542-561

33. Hussain A, Sial MS, Usman SM et al (2019) What factors affect patient satisfaction in public sector hospitals: evidence from an emerging economy. Int J Environ Res Public Health 16(6):994

34. Kugbey N, Oppong Asante K, Meyer-Weitz A (2019) Doctorpatient relationship mediates the effects of shared decision making on health-related quality of life among women living with breast cancer. S Afr J Psychol 49(3):364-375
35. Sun N, Rau PP (2017) Barriers to improve physician-patient communication in a primary care setting: perspectives of Chinese physicians. Health Psychol Behav Med 5(1):166-176

36. Smith A, Smith A, Mitchell S et al (2016) Person-centered outcomes measurement: NIH-supported measurement systems to evaluate self-assessed health, functional performance, and symptomatic toxicity. Behav Med Pract Policy Res 6(3):470-474

37. Hall E, Hall E, Tam E et al (2019) Development and prospective evaluation of CAPLET, a cancer ambulatory patient physical function longitudinal evaluation tool for routine clinical practice. Support Care Cancer 27(2):521-530

Publisher's note Springer Nature remains neutral with regard to jurisdictional claims in published maps and institutional affiliations.

\section{Authors and Affiliations}

\section{Yin Ting Cheung ${ }^{1} \cdot$ Alexandre Chan $^{2} \cdot$ Andreas Charalambous $^{3,4} \cdot$ H. S. Darling ${ }^{5} \cdot$ Lawson Eng $^{6} \cdot$ Lisa Grech $^{7,8,9,10}$. Corina J. G. van den Hurk ${ }^{11}$. Deborah Kirk ${ }^{12}$. Sandra A. Mitchell ${ }^{13}$. Dagmara Poprawski ${ }^{14}$. Elke Rammant ${ }^{15}$. Imogen Ramsey ${ }^{16} \cdot$ Margaret I. Fitch $^{17} \cdot$ Raymond J. Chan ${ }^{18} \cdot$ on behalf of the Multinational Association for Supportive Care in Cancer Survivorship Study Group.}

1 School of Pharmacy, Faculty of Medicine, The Chinese University of Hong Kong, Hong Kong SAR, China

2 School of Pharmacy \& Pharmaceutical Sciences, Department of Clinical Pharmacy Practice, University of California, Irvine, LA, USA

3 Department of Nursing, Cyprus University of Technology, Limassol, Cyprus

4 Department of Nursing, University of Turku, Turku, Finland

5 Department of Medical Oncology, Command Hospital Air Force, Bangalore, India

6 Division of Medical Oncology and Hematology, Department of Medicine, Princess Margaret Cancer Centre/University Health Network, University of Toronto, Toronto, Canada

7 School of Clinical Sciences, Faculty of Medicine, Nursing and Health Sciences, Medicine Monash Health, Monash University, Melbourne, Australia

8 Melbourne School of Psychological Sciences, University of Melbourne, Melbourne, Australia

9 Health Sciences, Swinburne University, Melbourne, Australia
10 Department of Cancer Experiences Research, Peter MacCallum Cancer Centre, Melbourne, Australia

11 R\&D Department, Netherlands Comprehensive Cancer Organisation, Utrecht, the Netherlands

12 School of Nursing and Midwifery, Edith Cowan University, Joondalup, WA, Australia

13 Division of Cancer Control and Population Sciences, National Cancer Institute, Bethesda, MD, USA

14 Department of Oncology, King Faisal Specialist Hospital \& Research Centre, Riyadh, Kingdom of Saudi Arabia

15 Department of Human Structure and Repair, Ghent University, Ghent, Belgium

16 Rosemary Bryant AO Research Centre, Clinical and Health Sciences, University of South Australia, Adelaide, Australia

17 Bloomberg Faculty of Nursing, University of Toronto, 207 Chisholm Ave, Toronto, ON, Canada

18 Caring Futures Institute, College of Nursing and Health Sciences, Flinders University, Bedford Park, South Australia 5042, Australia 